Cahiers de philosophie de l'université de

\title{
Introduction à la philosophie religieuse de Vladimir Soloviev
}

\section{Anastasia Yastrebtseva}

\section{(2) OpenEdition}

1 Journals

Édition électronique

URL : https://journals.openedition.org/cpuc/953

DOI : $10.4000 /$ cpuc. 953

ISSN : 2677-6529

Éditeur

Presses universitaires de Caen

\section{Édition imprimée}

Date de publication : 31 octobre 2011

Pagination : 47-61

ISBN : 978-2-84133-385-1

ISSN : $1282-6545$

\section{Référence électronique}

Anastasia Yastrebtseva, «Introduction à la philosophie religieuse de Vladimir Soloviev », Cahiers de philosophie de l'université de Caen [En ligne], 48 | 2011, mis en ligne le 01 septembre 2020, consulté le 03 février 2023. URL : http://journals.openedition.org/cpuc/953; DOI : https://doi.org/10.4000/cpuc. 953

\section{(c) (†) (8)}

Creative Commons - Attribution - Pas d'Utilisation Commerciale 4.0 International - CC BY-NC 4.0 https://creativecommons.org/licenses/by-nc/4.0/ 


\section{Introduction à la philosophie religieuse de Vladimir Soloviev}

$\mathrm{V}$ LAdimir Soloviev (1853-1900) est l'un des plus remarquables philosophes et poètes symbolistes de l'Âge d'argent en Russie. Fils du célèbre historien russe Sergei Soloviev, sa mère appartenait à une ancienne grande famille ukrainienne; son ancêtre, connu dans la pensée russe, Gregori Skovoroda (1722-1794), reçut le surnom de «Socrate ukrainien » : à la fin du $\mathrm{XIX}^{\mathrm{e}}$ siècle, les intellectuels l'ont considéré comme le premier philosophe slave, et comme le père de la tradition philosophique nationale russe. On l'a appelé aussi le "Diogène russe», «notre» Pythagore et Xénophane, le "Lomonossov des steppes». Ce qui le rapproche de Socrate, ce sont des «discours amicaux» socratiques que Skovoroda tenait avec ses élèves, entretiens qui portaient sur la question de l'homme, l'éducation de la vertu à travers la connaissance de soi, la paix de l'âme et le bonheur qu'on peut acquérir en suivant sa propre nature intérieure. Le philosophe Skovoroda se présente cependant comme un phénomène périphérique du processus intellectuel en Europe du XVIII ${ }^{\mathrm{e}}$ siècle. Pour lui, la philosophie n'est pas une somme de connaissances, un système, mais c'est une voie, une recherche risquée durant laquelle la nature humaine se manifeste et son existence se transforme.

Au centre du système philosophique de Skovoroda, il y a la conception de «trois mondes": le macrocosme - c'est l'univers des êtres vivants en général; le microcosme - c'est le monde social et l'homme tel qu'il est; et enfin, le monde des symboles. Ces trois mondes sont composés de matière et de forme. La matière est une nature visible, tandis que la forme est invisible. Elles sont éternelles, et la dialectique de leur action réciproque se montre dans l'acte permanent de la création à partir du néant. Dans la lumière du Bien suprême, les idées se représentent comme des paradigmes pré-éternels des choses, en communion avec lesquels la matière reçoit son être. On le voit, il y a une forte présence du néoplatonisme dans la pensée de Gregori Skovoroda, comme ce sera également le cas chez Soloviev, Frank et Chestov. 
La philosophie russe des XIX ${ }^{\mathrm{e}}$ et $\mathrm{XX}^{\mathrm{e}}$ siècles, à laquelle appartient Vladimir Soloviev a subi une grande influence de la philosophie classique allemande, surtout celle de Schelling et Hegel ${ }^{1}$. Ces idées ont déterminé à leur tour non seulement le domaine de la philosophie mais aussi plus généralement toute la culture du siècle d'Argent, surtout la poésie des symbolistes et les recherches religieuses de l'intelligentsia russe, dite la «conscience religieuse nouvelle» (Dmitri Merejkovski, Nikolaj Berdiaev, Vassili Rozanov et d'autres). L'œuvre de Soloviev a contribué en grande partie au développement futur de la philosophie religieuse russe; Florensky, Frank, Chestov supposent la pensée de Soloviev.

Son projet général est consacré à la création du système de la connaissance intégrale grâce à la synthèse de la philosophie, de la religion et de la science. Cette idée est mise à la base de sa philosophie de l'Unité totale dont le but principal est de servir non seulement la science pure, mais aussi la communauté sociale. Autrement dit cette connaissance intégrale est destinée à répondre aux questions qui agitent les esprits, et elle ne doit pas être une connaissance foncièrement abstraite et intellectuelle. Cette connaissance intégrale vise à donner la réponse à la question du sens de la vie de l'homme, ainsi qu'à la question du but ultime et dernier du processus cosmique et historique. Sur cette approche est fondée la conviction de Soloviev en la réalité de l'universel. Cette idée est formée chez lui sous l'influence de Spinoza et des idéalistes allemands, surtout Hegel, qui ont proposé des systèmes monistes. Soloviev a emprunté la prémisse panthéistique au spinozisme et à l'idéalisme allemand qui constitue ensuite le fond de sa théorie de l'Unité totale dont l'idée est inspirée notamment par Schelling. C'est immédiatement à Schelling que remonte aussi sa conviction que la volonté est un commencement définitif de l'être. Enfin, c'est à Schelling que remonte aussi la représentation de Dieu souffrant et pris dans le développement historique. Cette idée a déterminé le caractère de l'historicisme de Soloviev.

Le système philosophique de Soloviev est construit, conformément au schème historique typique de la pensée du XIX ${ }^{\mathrm{e}}$ siècle, comme une histoire du développement de l'esprit, comme un processus théo-cosmo-historique. En aspirant à se distinguer du sécularisme de la philosophie européenne moderne, Soloviev n'a pas voulu revenir au christianisme historique comme

1. Vladimir Soloviev a soutenu en 1874 une thèse intitulée La crise de la philosophie européenne. Contre les positivistes. Il a eu plus tard un poste de maître de conférences à la chaire de Philosophie de l'université de Moscou, puis il est allé à Londres pour continuer ses recherches, où il a étudié la littérature mystique et gnostique de Jakob Böhme, de Paracelse et de Swedenborg. Il s'intéressait aussi à la Kabbale, à l'occultisme et au spiritisme. 
ce fut le cas dans l'Église orthodoxe et catholique. Son but a consisté en la justification du christianisme à travers la théosophie.

Soloviev a commencé sa réflexion par la critique de la pensée abstraite. À son avis, le fondement abstrait est nécessaire pour la vie de l'homme et de l'humanité en général. Cependant son absolutisation mène la philosophie à l'impasse, ce que Hegel a bien montré en réduisant les notions rationnelles aux fondements autodéveloppants, quand il parle de substance-sujet. Soloviev indique que le défaut principal du système hégélien est la transformation des notions logiques en quelque chose d'indépendant privé du sujet qui réfléchit à l'aide de ces définitions. De ce fait le système hégélien est fondé sur les relations, tandis que les participants à ces relations, sujets réels, ont disparu. Soloviev écrit dans Principes philosophiques de la connaissance intégrale (1877) que Hegel «ne reconnaît rien sauf les définitions purement logiques et relatives [...] toutes ses définitions logiques sont des prédicats sans sujets [...] dans cet état elles perdent toute netteté et deviennent instables, chacune d'elles passe librement à son opposé [...] $»^{2}$. Ainsi, Soloviev critique l'idéalisme abstrait de Hegel du point de vue du réalisme spirituel qui distingue le sujet qui réfléchit et ce à quoi il réfléchit ${ }^{3}$. Le véritable étant pour Soloviev n'est ni une construction rationnelle, ni la notion, ni la donnée empirique. Le vrai étant pour lui est une essence réelle de l'esprit, le sujet de la volonté, la force de l'être. L'étant ne peut pas être le prédicat, il n'est que le sujet. Par contre, l'être est le prédicat: «L'être est, cela veut dire qu'existe celui qui existe» ${ }^{4}$.

En vérité, il existe seulement des esprits et des âmes qui sont des porteurs de la volonté et de la force, ils sont cet étant tandis que le monde empirique donné dans le temps et dans l'espace n'est que le phénomène, et ne se caractérise que comme un être. Cette idée est empruntée évidemment à Kant et à Schopenhauer. En se référant à la distinction kantienne du phénomène et de la chose en soi, Soloviev voit l'essence de la chose en soi (dans ses termes - de l'étant) dans la volonté. L'étant premier et ultime est Dieu que Soloviev détermine comme le néant positif à la suite du néoplatonisme et de la Kabbale.

En définissant l'étant comme ce qui apparaît, et l'être comme le phénomène, Soloviev interprète la liaison entre Dieu et le monde comme celle entre essence et phénomène. Cela veut dire qu'entre le principe transcendant

2. V. Soloviev, Principes philosophiques de la connaissance intégrale, in Euvres, Moscou, Misl', 1988, 2 vol., t. II, p. 277.

3. Guy Planty-Bonjour a pu écrire en ce sens que «la philosophie soloviévienne n'est en rien redevable à la philosophie hégélienne» (Hegel et la pensée philosophique en Russie, La Haye, M. Nijhoff, 1974, p. 232).

4. V. Soloviev, Principes philosophiques de la connaissance intégrale, p. 219. 
le monde et le monde comme il est, il existe une relation de nécessité à saisir rationnellement. Cette interprétation panthéistique porte la marque spinoziste et hégélienne puisque la limite insurmontable entre l'être et le monde que la théologie chrétienne pensait entre le Créateur et la créature, est supprimée. Cependant ici on peut voir la contradiction entre le réalisme spirituel de Soloviev et sa méthode rationaliste: si l'étant est un être transcendant spirituel, nous ne pouvons rien savoir de celui-ci si ce n'est grâce à la relation dans laquelle il s'ouvre à nous, c'est-à-dire, la Révélation. Cela n'est pas accessible pour la connaissance purement rationnelle. Soloviev affirme que l'être incompréhensible pour la raison est l'objet de la méditation mystique, qu'il traite comme une intuition particulière. À la suite de Schelling et des romantiques, il rapproche l'intuition avec la capacité productive de l'imagination (de ce point de vue, il s'oppose à Kant), et la philosophie avec l'art, mais l'acte créateur il le traite par analogie avec la transe, avec l'état passif et celui de la méditation qui, de l'avis de plusieurs mystiques, rend possible la sortie vers le monde transcendant des esprits, inaccessible à la raison et à l'expérience empirique.

En rapprochant l'inspiration avec l'intuition intellectuelle à laquelle s'ouvrent des vérités éternelles, ou des idées, Soloviev considère l'état d'inspiration et extatique comme le début de la connaissance philosophique. Cette dernière est donc liée à l'art et en même temps à la vision mystique spirituelle. Il écrit:

L'influence que les êtres idéaux exercent sur nous, en produisant la connaissance intelligible (et la création) de leurs formes idéales et leurs idées, s'appelle l'inspiration. Cette influence nous met hors de notre centre naturel commun, nous dirige vers la sphère suprême, en produisant l'extase. Alors le commencement déterminant sans intermédiaire de la connaissance philosophique est une inspiration 5 .

En avouant la possibilité de l'influence immédiate des êtres transcendants sur l'homme, et en identifiant cette influence avec la méditation intellectuelle des idées, Soloviev abolit donc la limite entre la pensée rationnelle et l'expérience mystique ${ }^{6}$.

Dans les cours de Munich 1827-1828, Systèmes des époques du monde, Schelling avait essayé de mettre en évidence la notion de l'Unité totale qu'il

5. V. Soloviev, Principes philosophiques de la connaissance intégrale, p. 207.

6. La suppression de la différence entre l'intuition mystiquement traitée et la capacité productive de l'imagination produit une confusion de la fantaisie artistique avec la révélation religieuse, ainsi elle donne la possibilité à la compréhension occulte et magique de l'art - c'est une particularité de l'œuvre de Soloviev ainsi que des autres poètes symbolistes - Alexandre Blok, André Bely, Viatcheslav Ivanov, etc. 
fut obligé de corriger toujours en raison des accusations de Fichte et de Jacobi. Pour lui, l'Unité totale suppose que tout ce qui existe puise son être dans Dieu et représente la substance étendue qui existe en Dieu et à travers lui. Selon Schelling, c'est une idée fondamentale de chaque religion. En polémique avec Jacobi, Schelling défend sa conception en distinguant trois notions: le théisme, le panthéisme et le monothéisme. Refusant le théisme de Jacobi, et corrigeant le panthéisme de Spinoza, Schelling appelle sa propre philosophie un monothéisme qui a ses particularités. Rejetant l'idée de polythéisme de la pluralité des dieux, il parle de la pluralité d'un seul Dieu et traite son propre monothéisme comme le panthéisme sursubstantiel.

Suite à l'idée de Schelling, Soloviev comprend la vraie unité comme l'Un en pluralité. Dieu est pour lui cet Un, et tout ce qui est, est une manifestation de cet Un. Dans son ouvrage Principes philosophiques de la connaissance intégrale, Soloviev reproduit l'argumentation de Schelling. L'essence divine s'ouvre devant nous, dit Soloviev, à travers le sentiment. C'est pourquoi il n'est pas nécessaire d'avoir des preuves de l'existence de Dieu: il n'est pas possible de déduire logiquement sa Réalité de la raison pure, mais elle est donnée par l'acte de la foi. En concluant à l'aide de la foi, ou de la "perception religieuse», pour l'existence de Dieu, Soloviev essaie de déduire logiquement le sens de l'essence divine, même si cela est en contradiction avec sa propre idée que ce sens ne peut être donné qu'à travers l'expérience.

Soloviev qualifie le fondement divin d' «éternel unique total» ou d' "un et tout». Cela veut dire que toute l'essence du monde est en Dieu puisque l'Unité totale est une unité en pluralité. Selon Soloviev, l'Un est libre de tout, c'est pourquoi l'Un est défini négativement par rapport à l'autre. En même temps l'Un ne peut rien avoir hors de lui-même, c'est pourquoi l'Un doit être défini positivement par rapport à l'autre. Donc, dans l'Un sont unis deux contradictions, deux pôles, ou deux centres: le premier est une liberté de toutes les formes possibles, de toutes les manifestations; le second est une force qui produit l'être, c'est-à-dire la pluralité des formes. Le premier pôle est nommé «Un», et le second est nommé «Puissance de l'être» ou la «première matière» qui entre dans l'Absolu comme son autre, comme son premier substrat, ou le fondement de Dieu. À la suite de la philosophie schellingienne et schopenhauerienne, Soloviev définit la première matière comme force, intention, aspiration - ce qui remonte à la conception de Jakob Böhme de la «nature obscure» en Dieu et de la profondeur inconsciente de Dieu, de laquelle découle le principe du Mal. L'indissolubilité de ces deux pôles de l'être en tant qu'être signifie que l'Absolu s'accomplit dans la matière, et la matière à son tour se montre comme une idée, comme une forme réalisée. Deux pôles sont liés éternellement et chacun d'eux est né de l'autre. 
Dans son œuvre Critiques des principes abstraits (1877-1880) Soloviev décrit le deuxième pôle de l'Unité totale qu'il appelle la première matière qui est, selon lui, un Un total toujours devenant à la différence du premier pôle qu'il appelle une essence de l'Un total. La thèse principale considère que l'absolu ne peut exister autrement que ce qui est accompli dans son autre. Cette compréhension de la relation entre le Dieu et le monde diffère de l'idée chrétienne de la création du monde. L'Un total qui est toujours dans le processus de devenir est l'âme du monde qui est à la base de tout le processus mondial, mais c'est seulement en l'homme que cet Un reçoit sa propre actualité intérieure. L'élément divin de l'âme mondiale, ou l'Unité totale, n'existe dans le monde préhumain et naturel que potentiellement. C'est seulement dans l'homme qu'il reçoit son actualité bien qu'il se montre d'abord à la façon idéale dans la conscience comme le but et la norme de l'activité humaine. L'acquisition de ce but est une tâche de l'histoire mondiale entendue comme le processus divino-humain.

Dans ses Leçons sur la divino-humanité (1880-1881), Soloviev traduit le processus de l'autodédoublement de l'Absolu dans le langage de la théologie chrétienne en donnant sa propre interprétation du dogme de la Trinité. Il distingue Dieu comme un Être Absolu de son idée. Cette dernière se présente comme Dieu-fils, ou Logos. Ce contenu se manifeste à l'âme du monde, que Soloviev appelle Sophia, qui présente la troisième image de la Trinité divine - le Saint Esprit. Soloviev écrit:

Et de même que l'Étant, en se distinguant de son idée, ne fait en même temps qu'un avec elle, de même le Logos, en se distinguant de la Sophia, est intimement lié à elle. La Sophia est le corps divin, la matière de la Divinité pénétrée par le principe de l'unité divine. Le Christ, en tant qu'organisme divin intégral qui réalise en soi ou porte cette unité - organisme tout à la fois universel et individuel -, est en même temps le Logos et la Sophia ${ }^{7}$.

Ici nous voyons un certain changement dans les présentations de Soloviev puisqu'il distingue maintenant en Dieu la double unité, à savoir: l'unité active du Logos créatif divin et l'unité créée et accomplie.

L'unité active est l'âme du monde en Dieu, tandis que l'unité créée est son corps organique. Dans le Christ ces deux unités sont présentées: la première est Dieu Christ, la force active, Logos, et la seconde unité créée désignée par le nom de Sophia, est le commencement de l'humanité.

7. V. Soloviev, Leçons sur la divino-humanité, in CEuvres, t. II, p. 108; traduction française: V. Soloviev, Leçons sur la divino-humanité, Paris, Cerf, 1991 (trad. par B. Marchadier), p. 116. 
L'humanité parfaite, ce n'est pas l'homme naturel comme phénomène, ce n'est pas l'être singulier empirique, aussi Sophia n'est pas l'humanité comme elle est figurée sur la terre, mais l'humanité comme idée éternelle. Cette idée éternelle de l'humanité est, selon Soloviev, Sophia - Féminité éternelle qui se trouve éternellement dans l'être divin.

Alors, l'idée de divino-humanité est liée étroitement chez Soloviev à sa conception de la sophiologie. Cependant il faut remarquer qu'ici deux réalités différentes auront lieu: l'idée de l'homme dans l'esprit divin se mêle invisiblement avec l'homme comme créature de Dieu. La limite entre éternel et temporel, transcendant et immanent, Créateur et créature devient assez transparente, peut-être disparaît-elle complètement. De ce fait, toute une actualité perd sa réalité et se transforme dans le monde trompeur, illusoire qui cache la vraie réalité. Mais dans ce cas, toute l'activité humaine et toute l'histoire humaine dans ce monde illusoire doivent être considérées soit comme le déploiement nécessaire du contenu de l'idée divine de l'homme, soit comme une agitation sans but et dénuée de sens.

Avec l'absence de limite claire entre le Créateur éternel et sa créature, le panthéisme change donc le dogme chrétien. Dieu ne crée pas librement le monde et l'homme, puisque l'homme empirique n'est que le phénomène nécessaire dans le temps de l'homme éternel considéré comme une idée divine. En conséquence, l'homme empirique est aussi privé de sa liberté, il n'est pas la source réelle de sa propre activité.

L'idée de la divino-humanité éternelle est issue de l'intention de Soloviev de réfléchir philosophiquement sur le dogme chrétien de l'incarnation: le Christ est un Logos incarné, car il a pris l'image de l'homme, la deuxième personne de la Trinité. Si on essaie de donner un traitement rationnel au mystère de l'incarnation du fondement divin, cela mène à l'idée de l'existence initiale de l'humanité idéale en Dieu. L'illusionnisme symbolique de la tradition romantique, surtout de Schopenhauer, que suit Soloviev, l'a mené à une idée précise, à savoir: c'est seulement à partir de la coéternité de l'homme à Dieu qu'on peut parler de l'immortalité humaine et de sa liberté. À ce propos Soloviev écrit:

En représentant l'homme comme un être créé ex nihilo dans le temps, et donc comme une sorte de créature contingente pour Dieu - car on suppose alors que Dieu peut aussi exister sans l'homme, et que c'est ce qui s'est passé avant la création de l'homme -, en présentant, dis-je, comme un être absolument déterminé par l'arbitraire divin et par conséquent absolument passif par rapport à Dieu, nous ne laissons aucune place à sa liberté ${ }^{8}$.

8. Ibid., p. 120 ; trad. p. 127-128. 
Soloviev rejette donc sciemment le dogme chrétien de la création qui ne dépouille pas du tout l'homme de l'immortalité et de la liberté. La légende biblique de la chute atteste déjà que l'homme est créé libre et peut utiliser sa liberté pour le Bien ou pour le Mal. Que l'homme et Dieu soient coéternels et que Dieu ne puisse pas exister sans l'homme, ces convictions de Soloviev sont dictées par son idée issue de l'idéalisme allemand ainsi que par des conceptions gnostiques et mystiques de l'absence de différence ontologique entre l'homme et le Dieu.

Il ne paraît pas étonnant que, dans le cadre de telles prémisses, Soloviev se heurte au problème de l'immortalité de l'âme humaine individuelle. L'être qui commence par la naissance physique doit se terminer par la mort physique. Le philosophe estime que l'existence interminable après la mort n'est aucunement liée du point de vue logique à la nullité avant la naissance. Ce n'est pas l'homme comme être individuel qui est immortel, mais c'est son essence éternelle intelligible. Selon Soloviev, cette essence n'est pas créée par Dieu, mais l'Homme éternel, ou l'Humanité idéale, est une individualité universelle, la divino-humanité et la divino-matière.

Notre réalité où les gens sont des individus, est, selon Soloviev, illusoire et non véritable à cause du principe de l'individualisation qui est à la base de l'égoïsme de chaque être et qui se manifeste dans toutes ses oppositions à l'autre et dans son aliénation. C'est la raison pour laquelle Soloviev dit que l'égoïsme est le Mal fondamental de tout ce qui existe. Le Mal, ou le "péché de l'individualisation», engendre l'être extérieur matériel, existant dans les conditions de l'isolement et de l'hostilité. Le mal et la souffrance sont les états de l'existence individuelle, mais si l'individualité est leur source, comment est-il possible de parler de l'immortalité individuelle? On ne peut trouver le Salut que dans la libération de l'existence individuelle, et jamais dans sa continuation éternelle. Nous voyons ici que la philosophie de Soloviev tend vers l'impersonnalisme. Le péché de l'individualisation, selon Soloviev, a ses racines en Dieu: "La cause efficiente du mal ne peut être l'être individuel dans sa manifestation naturelle déjà conditionnée, mais l'être dans son essence éternelle absolue, dont relève sa volonté initiale et immédiate " ${ }^{9}$. Le commencement du Mal n'est donc pas physique, mais métaphysique. C'est pourquoi chaque être naît dans les conditions du Mal, en choisissant l'égoïsme avant son arrivée au monde quand il n'y a pas encore une possibilité de choix conscient et libre.

Soloviev croit que la source du Mal dans le monde n'est pas dans la chute de l'homme issue de sa liberté, mais est au commencement dans l'Unité

9. V. Soloviev, Leçons sur la divino-humanité, p. 126; trad. p. 133. 
divine totale elle-même. Pendant que tous les êtres sont potentiellement en Dieu, et comme les esprits purs sont en unité avec la volonté divine, ils ne peuvent pas influer sur Dieu de l'extérieur parce qu'il leur faudrait avoir une existence séparée. Mais pour Dieu il est nécessaire que «les êtres multiples reçoivent leur individualité propre et réelle, sinon la force de l'unité divine ou de l'amour n'aura pas d'objet auquel se manifester ou se révéler dans toute sa plénitude [...]. Étant "libre d'envie" ${ }^{10}$, c'est-à-dire d'exclusivisme, il veut leur propre vie réelle " ${ }^{11}$. C'est pourquoi Dieu même crée leur être indépendant (de ce point de vue il engendre le Mal autant que le Bien), et ils peuvent maintenant influer sur lui puisqu'ils deviennent alors non pas seulement des êtres idéaux, des esprits, dont la vie consiste en la contemplation de Dieu, mais des êtres vivants et désirant avec une volonté autonome et réelle.

Cela peut donner l'impression que Soloviev trahit sa propre conception en reconnaissant la pluralité des substances-âmes indépendantes. Cependant il explique de quelle vie isolée de l'être indépendant il s'agit ici: "Cette seconde unité produite, s'opposant à l'unité première du Logos divin, c'est, nous le savons, l'âme du monde ou l'humanité idéale (Sophia), qui contient en soi et relie tous les êtres vivants particuliers, ou âmes ${ }^{12}$. Donc, il ne s'agit pas des êtres vivants particuliers mais de l'âme du monde qui possède l'unité substantielle. C'est elle qui reçoit l'indépendance, elle seule est un sujet libre et fait un acte de la chute de Dieu dont le sens est une intention de posséder toute la plénitude de l'être, c'est-à-dire de s'affirmer hors de Dieu. De ce fait, écrit Soloviev: l'âme du monde

[...] abandonne nécessairement sa position centrale, tombe du centre uni-total de l'être divin à la périphérie multiple de la création, perdant sa liberté et son pouvoir sur cette création [...]. Quand l'âme du monde cesse d'unir toutes choses, celles-ci perdent leur lien commun, l'unité du cosmos se désintègre en une multitude d'éléments séparés, et l'organisme universel se transforme en un ensemble mécanique d'atomes ${ }^{13}$.

Sophia chez Soloviev apparaît comme un personnage central du processus théocosmique. Sa nature est profondément ambiguë. D’un côté, elle est le principe féminin éternel de Dieu, l'âme du monde, le corps du Christ,

10. Cette expression vient de Platon, notamment du Phèdre (244a) et du Timée (29e) : la divinité qui par essence est bonne ne peut éprouver de jalousie et donc garder pour soi ce qu'elle pourrait communiquer et donner; Plotin reprend cette doctrine (voir traité Contre les gnostiques, II, 9 [33], 17, in Ennéades).

11. V. Soloviev, Leçons sur la divino-humanité, p. 128; trad. p. 136.

12. Ibid., p. 131 ; trad. p. 138.

13. Ibid., p. 132 ; trad. p. 139-140. 
l'humanité idéale, grâce à laquelle Dieu se manifeste comme une force active et vivante, comme le Saint Esprit. D’un autre côté, Sophia est un principe unifiant du monde créé, et elle se montre comme l'âme vivante de tous les êtres créés et séparés, ou comme une «humanité initiale»:

Étant une réalisation du fondement divin, son image et son simulacre, l'humanité initiale, ou l'âme du monde, est à la fois l'Un et Tout; elle occupe une place intermédiaire dans une pluralité des êtres vivants [...] et l'unité inconditionnée de Dieu [...]. Elle est comme un point crucial vivant ou une âme de tous les êtres, et en même temps la forme réelle de Dieu, le sujet vrai de l'être créé et l'objet réel de l'action divine [...] toute une humanité unie, ou l'âme du monde, est une essence ambiguë; concluant le commencement divin et le monde créé, elle ne se détermine exclusivement ni par l'un, ni par l'autre, et en conséquent elle est libre $[\ldots]^{14}$.

Quand Sophia est déterminée par le Logos divin, elle porte le principe divin à l'intérieur d'elle-même et unit tout ce qui existe. Mais elle possède sa propre volonté, et quand elle décide d'obtenir tout parce que c'est sa propre volonté, mais pas celle de Dieu, elle se distingue de lui et s'affirme librement. Dans cet état de détachement de Dieu, Sophia s'approprie les caractéristiques démoniaques. Insistant sur sa particularité, Sophia devient le principe du Mal. L’organisme mondial se multiplie en êtres séparés égoïstes. Soloviev développe une idée des gnostiques antiques pour qui l'évasion hors du Mal et de la guerre est un retour de l'âme détachée de l'unité de l'être divin.

Cependant Soloviev a très bien compris qu'en unifiant en Sophia les deux principes - divin et créé - il était obligé d'avouer que Dieu est coupable aussi dans l'apparition du Mal et du chaos dans le monde. Ici on peut voir l'influence du panthéisme schellingien. Il essaie d'un côté de représenter le péché et la mort comme des faits de l'actualité hors Dieu; la chute de l'âme du monde de Dieu est présentée comme une catastrophe intérieure ayant lieu dans le monde inconditionné et certain. Sophia est une Sagesse divine, elle appartient au monde divin tandis que l'âme du monde est un principe du monde créé. Afin de surmonter cette difficulté, Soloviev, dans La Russie et l'Église universelle $(1889)^{15}$, distingue Sophia et l'âme du monde qui est un opposé par rapport à Sophia. L'âme du monde est maintenant une source du Mal et du Chaos, tandis que Sophia, selon Soloviev, est « un être rayonnant du ciel séparé des ténèbres de la matière terrestre ${ }^{16}$.

14. V. Soloviev, Leçons sur la divino-humanité, p. 131; trad. p. 138.

15. L'œuvre originale fut publiée en français pour éviter la censure tsariste. Voir: V. Soloviev, La Russie et l'Église universelle, Paris, A. Savine, 1889; rééd. Lausanne, l'Âge d'homme, 1978.

16. V. Soloviev, La Russie et l'Église universelle, Moscou, Pout, 1911, p. 347. 
Cela étant, Soloviev n'a pas pu refuser pleinement le rapprochement de Sophia et de l'âme du monde. C'est pourquoi il appelle la seconde une «mère du chaos existant hors de Dieu ${ }^{17}$ et il la distingue du chaos lui-même. Le sens du processus mondial se représente donc comme une lutte entre le Logos divin et le principe infernal pour le pouvoir sur l'âme du monde. Cette lutte doit se terminer par un rattachement avec Dieu de l'âme du monde séparée et un rétablissement de l'Unité totale divine. Cette fin détermine l'évolution de la nature et de l'histoire. La fin du processus cosmique est une naissance de l'homme naturel après quoi vient le processus historique préparant la naissance de l'homme selon l'esprit.

La position de Soloviev correspond sur ce point au déterminisme téléologique commun au XIX ${ }^{e}$ siècle d'où vient non seulement l'optimisme mais aussi l'utopisme de l'historiosophie de Soloviev. Son idée principale est que le processus historique doit mener par une nécessité intérieure au triomphe du Bien. Il est convaincu que le développement historique mènera au triomphe de l'unité et de l'amour sur la désagrégation et l'animosité. Puisque cette désagrégation est un moment nécessaire du processus mondial initié par Dieu, la chute de l'âme du monde est un passage inévitable dans l'histoire du monde qui annonce son rattachement futur avec Dieu.

Il faut remarquer aussi qu'à la différence du christianisme traditionnel, ou de l'idée de la dimension fondamentale du péché, Soloviev s'intéresse à la sphère mystique, aux péripéties de la vie de Sophia considérée comme une humanité idéale, à sa chute et à son salut, à son rattachement à Dieu, et en conséquence il ne voit pas la réalité du monde à travers lequel il contemple le mystère divin et, parfois, il occulte la variété empirique du monde.

Soloviev propose une historiosophie originale qui est sa tentative de comprendre l'histoire mondiale comme une «série d'actes libres» en voie vers le rétablissement de l'unité divino-humaine, vers le dialogue entre Sophia et Logos, qui se montre dans le développement spirituel, religieux, moral et artistique. Au début, au premier degré de la révélation naturelle et immédiate, l'humanité a conçu Dieu comme un être naturel. Telles sont les croyances païennes de l'Antiquité. Au second degré, Dieu s'ouvre aux gens comme un commencement opposé à la nature, comme une transcendance et comme un néant. Telles sont les révélations négatives des religions ascétiques et pessimistes de l'Orient. Elles se trouveraient aussi dans la religion de l'Ancien Testament où Dieu est personnel, ainsi que dans la conception de Logos développée dans la philosophie grecque; enfin l'humanité a reçu

17. Ibid. 
la révélation positive de la Vérité dans le christianisme, à savoir dans la personnalité divino-humaine du Christ. Selon Soloviev, le Christ est une synthèse du commencement religieux contemplatif propre aux religions orientales, avec celui de la qualité personnelle, humaine, active, développé au sein de la culture européenne.

Cependant le schisme des Églises orientales et occidentales a marqué une nouvelle époque de la désagrégation du monde chrétien. C'était un signe, selon Soloviev, de l'imperfection du christianisme historique. L'unité spirituelle s'est alors divisée à nouveau en deux aspects. À l'Est c'est le monisme qui a gagné, c'est-à-dire la domination du principe surindividuel divin qui ne laisse pas de place pour la liberté et l'indépendance de l'homme. À l'Ouest, au contraire, c'est l'individualisme hypertrophié et la liberté dans son sens négatif qui se sont développés, ce qu'on peut décrire comme une libération loin de l'unité et, finalement, la domination du chaos.

Dans les années 1830-1840 en Russie s'est formé un nouveau courant intellectuel - le slavophilisme - qui est devenu l'alternative à la propagation large dans la société des théories rationalistes, avant tout des idées des Lumières. La franc-maçonnerie russe du XVIII ${ }^{\mathrm{e}}$ siècle, le mysticisme et puis les efforts des schellingiens russes ont été orientés vers l'évincement de l'influence de la philosophie des Lumières, ce qui a provoqué le basculement de la pensée russe vers la philosophie nouvelle allemande, surtout vers Schelling et vers Hegel. Le but principal des slavophiles (Alexis Khomiakov, Ivan Kiréievski, Ivan Aksakov) a consisté à réveiller la conscience nationale. Selon leur conviction, la mission historique de la Russie, son authenticité consiste dans le Salut du monde, c'est pourquoi elle doit chercher sa propre voie de développement. Cette idée les a opposés aux occidentalistes (Piotr Tchaadaïev, Vissarion Belinski, Alexandre Herzen) qui considéraient le patriotisme bien compris comme impliquant l'européanisation de la Russie.

À la suite des slavophiles, Soloviev a prononcé une condamnation des vices de la civilisation occidentale. Il s'est trouvé que la vérité est en fait ambiguë: le monde oriental affirme le "Dieu inhumain" tandis que le monde occidental affirme l'«homme non-divin». La question traite de la possibilité de surmonter cette rupture fatale. Pour Soloviev le rôle principal pour trouver une solution appartient à un peuple libre, au caractère national du peuple russe. D'où vient que, selon lui, la Russie a une mission religieuse toute particulière: réunir les éléments séparés de l'histoire humaine et accomplir le dernier acte du drame de la divino-humanité.

La conception théosophique de Soloviev s'est formée encore dans les années 1870. Dans les années 1880, elle s'est transformée en une théorie utopiste, celle de la théocratie future universelle. Dans les Principes philosophiques de la connaissance intégrale (1877) Soloviev parle de la théocratie 
libre qu'il identifie à la société intégrale opposée à la société contemporaine atomistique et séparée. Dans la théocratie libre, écrit Soloviev, la société spirituelle, autrement dit l'Église, constitue un organisme intégral en union intérieure libre avec les sphères politiques et économiques de la vie.

Son ouvrage L'histoire et l'avenir de la théocratie (1885-1887) a pour sous-titre: «L'étude de la voie historique universelle vers la vie véritable». Celui-ci explique l'intention de Soloviev de «justifier la foi de nos pères en l'élevant à un nouveau degré de la conscience rationnelle; montrer que cette antique foi, délivrée des chaînes du particularisme local et de l'égoïsme national, coïncide avec la vérité éternelle et universelle ${ }^{18}$.

Parlant de l'amour-propre populaire Soloviev indique que l'acquisition et la réalisation de la vérité dans la vie n'est possible qu'avec le rétablissement de l'union détruite des Églises chrétiennes orientale et occidentale. Soloviev connaît très bien la critique slavophile par rapport à l'Église catholique. Il reconnaît que les slavophiles expriment la conscience de l'Église orthodoxe et du peuple russe en général: «[...] le catholicisme et tout rapprochement avec lui sont étrangers à notre connaissance nationale [...]». Cependant il dit aussi que c'est sur cette voie seulement qu'il est possible de réaliser la mission historique des peuples slaves: «Dieu n'a pas créé la Russie aussi grande et puissante pour des affaires légères et simples. Bien qu'il soit difficile d'unir les églises, cela n'empêche pas d'en faire notre but vital et notre mission historique globale $»^{19}$.

La dimension utopique de sa conception du monde encourage Soloviev à soutenir le développement de l'idée de la théocratie totale. Mais avant de parler des principes de la théocratie totale future, Soloviev analyse le développement de l'idée théocratique dans l'histoire des juifs. Il considère l'Ancien Testament du point de vue de la genèse de la forme nationale de la théocratie qui serait un précurseur de la théocratie mondiale. Selon lui, cette dernière pourra être réalisée par des peuples chrétiens sous l'égide du pouvoir du Tsar russe et sous celle du pouvoir spirituel du Pontife de Rome. En fait, cette idée de la théocratie mondiale illustre la possibilité de la réalisation du règne de Dieu sur la terre. Dans son article Le judaïsme et la question chrétienne, Soloviev écrit que les chrétiens ainsi que les juifs ne tendent pas seulement au renouvellement de l'esprit humain mais aussi à celui du ciel et de la terre. C'est là où habite la vérité. Le règne de Dieu est à l'intérieur, dans l'esprit, mais aussi à l'extérieur, dans la puissance politique:

18. V. Soloviev, Histoire et avenir de la théocratie, in Euvres, Saint-Pétersbourg, Obtchestvennaya polza, 1904, t. IV, p. 214; traduction française: Paris, Cujas, 2008, p. 19.

19. Ibid., p. $235-236$. 
il s'agit d'une véritable théocratie ${ }^{20}$. Le précepte principal de la théocratie est une supériorité du pouvoir spirituel sur le pouvoir laïc. La société civique doit se transformer conformément au règne de l'Église où, à côté de la loi et de la justice (principes de l'État), règne le fondement de l'amour.

Dans cette conception théocratique de Soloviev, on voit donc la limite dépassée entre temporel et éternel, terrestre et céleste, et c'est là une caractéristique générale de la philosophie de Soloviev. Seulement à la fin de sa vie il a renoncé à son utopie théocratique, surtout dans l'ouvrage Trois entretiens sur la guerre, la morale et la religion ${ }^{21}$.

Étant désillusionné sur la possibilité de réaliser son projet utopique de théocratie universelle ${ }^{22}$, Soloviev a changé d'opinion sur le statut de l'éthique. Si, dans ses premiers travaux, il soulignait la dépendance de l'éthique et de la métaphysique religieuse, plus tard le philosophe insiste sur l'autonomie de l'éthique puisqu'en créant la philosophie morale, la raison développe seulement sur le terrain de l'expérience l'idée du Bien qui lui appartient dès le début. Cependant, en étant autonome, cette éthique ne peut pas être distinguée absolument de la métaphysique et de la religion dans la mesure où seule l'idée du processus divino-humain et de la victoire de l'unité divine totale permet d'affirmer le fondement de la moralité, l'actualité du Bien surhumain. L'absence de ce fondement est, à son avis, une des faiblesses de l'éthique kantienne.

Dans La justification du bien, Soloviev critique aussi les autres conceptions morales diffusées à la fin du XIX ${ }^{\mathrm{e}}$ siècle, à savoir: l'immoralisme de Nietzsche ${ }^{23}$, la subjectivité morale de Léon Tolstoï et l'autoritarisme dans le traitement de la moralité. La plus forte critique de Soloviev est dirigée contre la tentative de Nietzsche de changer la moralité chrétienne pour le

20. V. Soloviev, Le judaïsme et la question chrétienne, in Euvres, Saint-Pétersbourg, 1904, t. IV, p. 140; traduction française: Paris, Desclée, 1992.

21. V. Soloviev, Trois entretiens sur la guerre, la morale et la religion [1899], Paris, Plon-Nourrit, 1916 (trad. par E. Tavernier).

22. Soloviev évoque cette désillusion dans l'introduction à sa traduction de Platon: «Avec l'accumulation de l'expérience, sans aucun changement en ce qui concerne l'essentiel de mes convictions, je doutais de plus en plus de l'utilité et du caractère réalisable des projets extérieurs auxquels j'ai consacré "mes meilleures années". Désespérer de cela signifiait retourner aux études philosophiques", Sobranie sotchinenii [Euvres complètes], Bruxelles, Jizn's Bogom, 1966, tome XII, p. 87; traduit et cité par Igor Sokologorsky dans son étude "Platon et le tournant des dernières années de Soloviev", Le temps philosophique [Paris X-Nanterre], $\mathrm{n}^{\circ}$ 10, 2000, Présences grecques dans la pensée russe, A. Charles-Saget (dir.), p. 95. L'auteur souligne le parallèle qu'établit Soloviev entre son propre renoncement aux projets «extérieurs » utopistes et celui de Platon après son échec politique avec Denys de Syracuse. On se tourne vers la spéculation métaphysique quand la praxis est décevante et nous fait comprendre que le mal dans le monde est indépassable.

23. Voir infra les deux articles de Soloviev traduits par A. Muller. 
culte païen de la puissance et de la beauté. Soloviev dit que le christianisme ne nie ni la puissance, ni la beauté, mais qu'elles y sont unies avec le Bien.

Cependant Soloviev considère aussi comme faux le point de vue contraire qui souligne le caractère seulement objectif de la moralité et voit son fondement dans l'autorité extérieure et dans les institutions sociales. Les adeptes de ce point de vue ne voient pas l'importance de la conscience et de l'activité de la personnalité. Ils sont convaincus que les formes historiques de cette vie sont elles-mêmes sages et bonnes et que l'homme ne doit que s'incliner devant elles avec vénération.

Soloviev croit que la synthèse de ces deux points de vue, le subjectif et l'objectif, est nécessaire, mais le philosophe accentue l'importance des formes objectives de la vie morale. Dans son œuvre La justification du bien il analyse profondément les sentiments, ou les sources de la moralité humaine - la honte, la pitié et la vénération. L'homme a honte de sa nature basse, surtout en ce qui concerne la honte sexuelle; la pitié, elle, est la compassion qu'il éprouve par rapport à tous les êtres vivants. En le poussant à l'altruisme, la pitié est à la base des liaisons sociales. Donc, la honte est individuelle tandis que la pitié est une pudeur collective. Enfin, le sens de la vénération devant le principe suprême est le fondement moral de la religion. On le voit, philosophie et religion sont selon Soloviev deux noms pour un même mouvement spirituel, celui par lequel l'esprit humain cherche à retrouver l’Unité perdue.

Anastasia Yastrebtseva

Université d'État, École des hautes études en sciences économiques (Moscou) 